\title{
Identificação de Sinais Precoces de Autismo Segundo um Protocolo de Observação Estruturada: um Estudo de Seguimento
}

\author{
Felipe Alckmin-Carvalho \\ Universidade de São Paulo \\ São Paulo, SP, Brasil \\ Maria Cristina Triguero Veloz Teixeira \\ Décio Brunoni \\ Vanessa Godoy Strauss \\ Cristiane Silvestre Paula \\ Universidade Presbiteriana Mackenzie \\ São Paulo, SP, Brasil
}

\begin{abstract}
RESUMO
Intervenções precoces, intensivas e de longo prazo estão associadas a impacto positivo no prognóstico de crianças com Transtornos do Espectro do Autismo-TEA. Os objetivos deste estudo foram: acompanhar crianças de 16-24 meses até atingirem três anos a fim de confirmar/refutar o diagnóstico de TEA e verificar se um protocolo estruturado de observação desenvolvido no Brasil seria capaz de discriminar crianças de alto/baixo risco para TEA. Instrumentos: Modified Checklist for Autism in Toddlers-M-CHAT, Autism Behavior Checklist-ABC, Protocolo de Observação Estruturada para Rastreamento de Autismo-OERA, Escalas Vineland e Bayley. Dos 11 casos acompanhados, nenhum confirmou diagnóstico de TEA de acordo com avaliação médica-DSM-IV. O OERA discriminou todos os casos negativos de TEA. Conclusões: verificou-se que M-CHAT foi sensível a atrasos no desenvolvimento, mas pouco específico para TEA sendo necessárias outras avaliações para o diagnóstico; OERA mostrou-se de baixo custo, bem aceito e importante para a discriminação entre atrasos de desenvolvimento e TEA.
\end{abstract}

Palavras-chave: Transtornos do espectro do autismo; Diagnóstico precoce; Desenvolvimento infantil.

\section{ABSTRACT}

Identification of Early Signs of Autism According to a Structured Observational Protocol: a Follow-up Study

Early, intensive and long-term interventions are associated with a positive impact on the prognosis of children with Autism Spectrum Disorders-ASD. The objectives of this study were to follow children with 16-24 months until three years of age in order to confirm/refute the diagnosis of ASD and verify if a structured observation protocol developed in Brazil would be able to discriminate children at high/low risk for ASD. Instruments: Modified Checklist for Autism in Toddlers-M-CHAT, Autism Behavior Checklist-ABC, Structured Observation for Autism Screening-OERA, Bayley and Vineland Scales. Of the 11 participants, none confirmed ASD diagnosis according to DSM-IV. The OERA was able to discriminate all negative cases of ASD. Conclusion: it was verified that M-CHAT was sensitive to identify developmental delays, but not specific regarding to ASD, being necessary other assessments to confirm the diagnosis; OERA was considered low cost, well accepted and important to discriminate developmental delays from ASD.

Keywords: Autism spectrum disorders; Early diagnosis; Child development.

\section{RESUMEN}

Identificación de Señales Tempranos de Autismo de Acuerdo con un Protocolo de Observación Estructurada: un Estudio de Seguimiento

Intervenciones tempranas intensivas y de largo plazo están asociadas a un impacto positivo en el pronóstico de niños con Trastornos del Espectro del Autismo (TEA). Los objetivos de este estudio fueron: acompañar niños de 16-24 meses hasta alcanzar tres años con la finalidad de confirmar o rechazar el diagnóstico de TEA y verificar si un protocolo estructurado de observación desarrollado en Brasil sería capaz de discriminar niños de alto/bajo riesgo para TEA. Instrumentos: Modified Checklist for Autism in Toddlers (M-CHAT), Autism Behavior Checklist (ABC), Observación Estructurada para Despistaje de Autismo (OERA), Escalas Vineland y Bayley. De los 11 casos acompañados, ninguno confirmó diagnóstico de TEA de acuerdo con la evaluación médica (DSM-IV). El protocolo OERA discriminó todos los casos negativos de TEA. Conclusión: se verificó que el M-CHAT fue sensible a atrasos en el desarrollo, pero poco específico para TEA siendo necesarias otras evaluaciones para el diagnóstico; OERA se mostró de bajo costo, bien acepto e importante para la discriminación entre atrasos del desarrollo y TEA.

Palabras clave: Trastornos del espectro del autismo; Diagnóstico precoz; Desarrollo infantil. 


\section{INTRODUÇÃO}

Os Transtornos do Espectro do Autismo (TEA) estão entre os problemas de saúde mental que mais prejudicam o desenvolvimento infantil. Trata-se de um transtorno que compromete, em diferentes níveis, duas áreas específicas do desenvolvimento: comunicaçãosocial e comportamento (American Psychiatric Association, 2013).

Pesquisas evidenciam que os primeiros sinais e sintomas de TEA começam a surgir entre seis e doze meses, tornando-se mais perceptíveis e estáveis entre os 18 e 24 meses, período em que já é possível fazer a identificação precoce dos casos (Belini \& Fernandes, 2007; Clifford, Young, \& Williamson, 2007; Ozonoff, 2008). Os sinais que podem ser notados precocemente são aqueles diretamente relacionados aos TEA, como prejuízo no contato visual e na atenção compartilhada, reduzido interesse social e em jogos de compartilhamento de prazer, modo de brincar incomum, alterações nos padrões comportamentais de respostas sensoriais (Ozonoff et al., 2010; Tamanaha, Perissinoto, \& Chiari, 2008), assim como outros sinais globais de atraso, incluindo irritabilidade, alterações no nível de atividade e déficits no desenvolvimento motor grosso (Cardoso \& Fernandes, 2006).

Estudos recentes apontam que quando o diagnóstico de TEA é realizado nos primeiros três anos de vida da criança, intervenções precoces intensivas e de longo prazo estão associadas a um impacto positivo no prognóstico, especialmente nas áreas de adaptação psicossocial e familiar, desempenho cognitivo, comportamento adaptativo, habilidades de comunicação e de interação social (Bosa, 2006; Carvalho et al., 2013; Eldevik et al., 2009). No entanto, mesmo com a constatação sobre a possibilidade de rastreamento do transtorno ainda na primeira infância e do impacto positivo no prognóstico quando há intervenções precoces, apenas uma minoria dos casos é diagnosticada antes do período pré-escolar (Bosa, 2006).

Instrumentos padronizados são de grande importância para a triagem e o diagnóstico de indivíduos com TEA, que é realizado basicamente por critérios clínicos (Teixeira et al., 2010). Dentre os instrumentos de triagem mais utilizados em pesquisas brasileiras, destacam-se: Modified Checklist for Autism in Toddlers (M-CHAT), Autism Behavior Checklist (ABC), Autism Screening Questionnaire (ASQ) e Childhood Autism Rating Scale (CARS), questionários destinados aos pais ou cuidadores, de fácil aplicação e baixo custo. Para o diagnóstico do transtorno, destacam-se a entrevista Autism Diagnostic Interview-Revised (ADI-R) e a
Autism Diagnostic Observation Schedule (ADOS), ambos considerados padrão ouro para o diagnóstico de TEA. Não há estudos sobre evidências de validade da ADOS, enquanto que um estudo piloto apontou ótimas propriedades psicométricas da versão brasileira da ADI-R (Becker et al., 2012).

Sabe-se que para a identificação de TEA é fundamental considerar o relato de múltiplos informantes, somado à observação direta dos comportamentos da criança (Ozonoff et al., 2008). Entre as ferramentas de observação do comportamento infantil, destaca-se a técnica de análise de filmagem da criança em situações padronizadas e eliciadoras de comportamentos apontados como marcadores para o transtorno (ex. prejuízos no contato visual, na orientação para o chamado ao próprio e em aspectos de receptividade) (Baranek, 1999). Uma vez que sinais de TEA já estavam presentes em gravações caseiras realizadas no primeiro ano de vida de crianças posteriormente diagnosticadas com TEA, a análise de vídeos em situações estruturadas tem sido utilizada como uma estratégia auxiliar eficaz para a avaliação do transtorno (Clifford, Young, \& Williamson, 2007). AADOS foi construída a partir desses preceitos, sendo atualmente o instrumento com melhores evidências para o estabelecimento de TEA, segundo a perspectiva de observação direta da criança (Gotham, Pickles, $\&$ Lord, 2009). Contudo, trata-se de uma medida de alto custo, que exige conhecimento clinico prévio e treinamento bastante complexo, sendo praticamente inviável para ser utilizado em larga escala. Assim, enquanto a ADOS é considerada o padrão ouro para o estabelecimento do diagnóstico de TEA, instrumentos de rastreamento, mais breves e simples são essenciais para a triagem de casos suspeitos.

Diversas pesquisas posteriores confirmaram que falhas na habilidade de orientar-se pelo próprio nome, por volta de um ano de idade, apresentam-se como um marcador precoce importante, associado ao desfecho positivo para TEA (Ozonoff et al., 2010). A orientação e sustentação da atenção para objetos, assim como particularidades na forma de brincar, exploração em menor frequência e menor flexibilidade de brinquedos e responsividade e comunicação social também têm sido relatadas como marcadores precoces associados aos desfechos de TEA (Kalb et al., 2010). Esses dados apontam para a necessidade de uma avaliação mais acurada do desenvolvimento infantil, especialmente nos casos em que as habilidades e comportamentos descritos estiverem deficitários ou prejudicados.

$\mathrm{Na}$ Europa, Estados Unidos e Japão os estudos apontam um progressivo aumento das taxas de prevalências de TEA na população, que segundo 
pesquisas recentes variam entre $0,6 \%$ e $1 \%$, sendo o transtorno muito mais prevalente do que se imaginava há algumas décadas (Fombonne, 2009). Estudos epidemiológicos de outras regiões do mundo fornecem dados menos robustos, mas que apontam na mesma direção (Elsabbagh et al., 2012). No Brasil, até o presente momento, foi realizado um único estudo (piloto) estimando a prevalência de TEA em 0,3\% (Paula, Ribeiro, Fombonne, \& Mercadante, 2011), portanto, novas pesquisas nacionais seriam de grande relevância no sentido de verificar de forma mais acurada a prevalência do transtorno na população brasileira (Bosa, 2006). A partir destes estudos, é possível estimar que aproximadamente 1,5 milhões de brasileiros tenham TEA (Paula et al., 2011), o que aponta para a relevância do tema, assim como para a necessidade de estudos que contribuam para a identificação de casos, a fim de consolidar conhecimentos que possam sustentar a elaboração de políticas públicas para assistir adequadamente essa demanda.

Paralelamente, uma recente revisão sistemática brasileira sobre TEA mostrou um aumento significativo da produção acadêmica sobre o tema, especificamente nos estados de São Paulo e Rio Grande do Sul. No entanto, a maior parte das publicações apresenta fragilidades metodológicas, como amostras reduzidas e intervenções sem controle de variáveis, enquanto que estudos sobre a identificação precoce de TEA incluindo dados de observação direta da criança e o seguimento de casos no decorrer do tempo são praticamente inexistentes (Teixeira, et al., 2010).

Considerando a relevância do assunto, o grande número de indivíduos com TEA e a necessidade de novas pesquisas em cenário nacional, os objetivos deste estudo foram: acompanhar crianças que tiveram sinais precoces de TEA rastreados, em uma pesquisa anterior, a fim de confirmar ou refutar o diagnóstico do transtorno e verificar se um protocolo estruturado de observação desenvolvido no Brasil seria capaz de discriminar crianças de alto e de baixo risco para TEA.

\section{MÉTODO}

Este projeto seguiu o desenho quantitativo observacional, em que um grupo de crianças de creches públicas do município Barueri-SP foi avaliado e acompanhado por um período de seis a nove meses.

Primeiramente faz-se necessário descrever brevemente uma pesquisa concluída em 2009 (Carvalho et al., 2013), a qual o estudo atual dá continuidade. O principal objetivo daquela pesquisa foi identificar sinais precoces de TEA em uma amostra de 104 crianças ingressantes em creches públicas de Barueri.
Para avaliação destes sintomas, foram utilizados o Modified Checklist for Autism in Toddlers (M-CHAT) (Robins, Fein, Barton, \& Green, 2001) e a Pictorial Infant Communication Scales (PICS) (Delgado, Mundy, \& Block, 2001), ambos preenchidos pelas cuidadoras das creches que as crianças frequentavam. O principal resultado da pesquisa foi a identificação de cinco crianças com idades entre 16 e 24 meses com suspeita de TEA (fase 1).

O presente estudo baseou-se numa reavaliação de parte da amostra anteriormente investigada, incluindo todas as crianças de alto risco e um grupo de crianças de baixo risco para TEA (fase 2 ). $\mathrm{Na} 2^{\mathrm{a}}$ fase, as crianças consideradas de alto risco para TEA (segundo M-CHAT aplicado na fase 1) foram pareadas com crianças que haviam sido consideradas de baixo risco para o transtorno e passaram por uma protocolo de avaliação que será descrito abaixo. Após um período de seis a nove meses todas as crianças já tinham completado três anos e foram novamente avaliadas pela mesma equipe de pesquisadores, compondo a $3^{\mathrm{a}}$ fase da pesquisa. $\mathrm{O}$ organograma abaixo apresenta as três fases do estudo com os correspondentes instrumentos de cada uma delas (Figura 1).

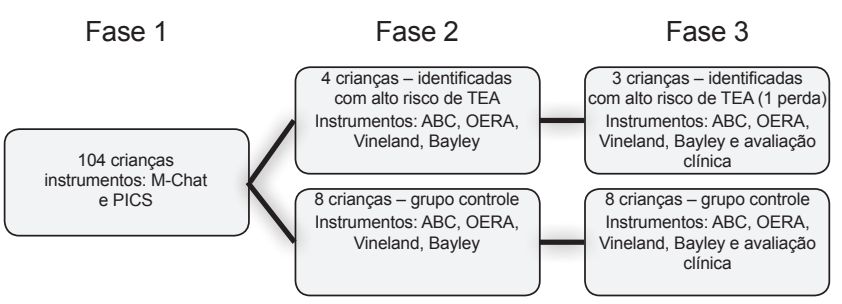

Figura 1. Organograma das atividades realizadas discriminadas por fases.

\section{Amostra}

O grupo de crianças que compõem a amostra do presente estudo é proveniente de cinco das 21 creches públicas do município de Barueri, que oferecem 700 vagas por ano para novas crianças, permitindo seu ingresso aos 12 meses de idade (Prefeitura Municipal de Barueri, 2012).

A amostra da segunda fase do estudo era inicialmente composta por cinco crianças consideradas de alto risco para TEA, provenientes das 104 avaliadas no estudo de 2009 rastreadas a partir da escala M-CHAT, no entanto, uma criança mudou de cidade e a mãe se recusou a participar das outras fases do estudo, sendo considerada uma perda. Assim, a amostra de crianças de alto risco para TEA (casos) passou a ser constituída de quatro sujeitos. Para formar o grupo 
controle, foram selecionadas oito crianças a partir dos 99 casos identificados como de baixo risco para TEA na fase 1 (dois controles para cada caso). A seleção do grupo controle foi feita por pareamento com os casos da seguinte forma: cada caso foi pareado com duas crianças do mesmo sexo, com idades aproximadas, que frequentavam as mesmas creches, mas que apresentavam baixo risco de serem diagnosticadas com TEA (crianças que não tiveram sinais precoces de TEA rastreados por meio do M-CHAT e da PICS na avaliação de 2009).

\section{Instrumentos}

\section{Fase 1}

Modified Checklist for Autism in Toddlers (M-CHAT): escala estruturada desenvolvida para rastreamento de sinais de TEA, contendo 23 questões do tipo sim/não, relacionadas à avaliação de Resposta Social (interesse em outras crianças e imitação) e Atenção Compartilhada (protodeclarativa e olhar monitorado). A escala foi desenvolvida para ser aplicada a pais de crianças com idades entre 16 e 30 meses no formato autoaplicável (Kleinman et al., 2008).

\section{Fase 2}

(a) Autism Behavior Checklist (ABC): Instrumento desenvolvido para o rastreamento de sinais precoces de TEA, destinado aos pais e cuidadores. Abarca itens que investigam déficits em três domínios: sociabilidade, comunicação e comportamentos estereotipados e restritivos. Possui 57 itens que avaliam indivíduos com idades entre 18 e 35 meses nas seguintes áreas: sensoriais, relacionais, imagem corporal, linguagem, interação social e autocuidado (Krug, Arick, \& Almond, 1980).

(b) Protocolo de Observação Estruturada para Rastreamento de Autismo (OERA): desenvolvido por nossa equipe com base nos principais sinais precoces de TEA relatados na literatura (Baranek, 1999; Belini \& Fernandes, 2007; Clifford et al., 2007; Ozonoff et al., 2008) e nas provas da The Autism Diagnostic Observation Schedule-Generic (ADOS-G), instrumento internacional considerado padrão-ouro para o diagnóstico de TEA (DiLavore, Lord, \& Rutter, 1995). A partir deste material, foram selecionadas as provas consideradas as mais relevantes teoricamente, ao mesmo tempo em que eliciavam comportamentos observáveis e que não necessitavam muito conhecimento clínico do avaliador, facilitando o uso do OERA por profissionais pouco especializados. A OERA é composta por oito provas que eliciam comportamentos especificamente relacionados aos sintomas centrais de TEA. Durante 10 a 15 minutos o avaliador interage com a criança seguindo as seguintes provas estruturadas que são filmadas e posteriormente pontuadas por especialistas em TEA: (1) resposta ao nome; (2) iniciação da atenção compartilhada; (3) resposta à atenção compartilhada; (4) imitação funcional e simbólica; (5) resposta ao sorriso; (6) nomear figuras; (7) compartilhamento de prazer em jogo de bolha de sabão e (8) apontar para objeto. Durante toda a prova, os avaliadores também observam, registram e pontuam o perfil comportamental da criança considerando a presença/ ausência de outros 14 itens englobando os seguintes aspectos: (1) linguagem e comunicação (6 itens, como por ex: quantidade e qualidade da vocalização dirigida ao outro; presença de ecolalia); (2) interação social recíproca (4 itens, como por ex: ausência de sorriso social espontâneo; adequação e intencionalidade da expressão facial); e (3) estereotipias motoras, maneirismos, comportamentos de autoagressão e comportamentos estereotipados (4 itens). Cada item deve ser pontuado como SIM (para comportamento presente $=1$ ponto) ou NÃO (para comportamento ausente $=$ zero pontos). Deste modo, a pontuação total do OERA pode variar de zero e 22 pontos, sendo 8 pontos relativos as provas estruturadas e 14 pontos referentes a avaliação global do perfil comportamental durante toda a avaliação. Este protocolo permite estabelecer o desfecho de "provável caso de TEA" ou de criança com desenvolvimento típico.

(c) Escala Comportamento Adaptativo de Vineland: tem como objetivo avaliar a independência pessoal e social das crianças e adolescentes, desde o nascimento até 18 anos de idade, descrevendo, portanto, seu comportamento adaptativo. A Vineland tem três versões e no presente estudo foi utilizada a Versão Entrevista mais Formulário Expandido, que é baseada em uma entrevista com pais e dura aproximadamente de 20 minutos (Sparrow \& Cicchetti, 1985). A avaliação do nível de comportamento adaptativo das crianças com TEA foi incluída no presente estudo, pois devido a grande variabilidade nos quadros de TEA, esta tem sido considerada um dos melhores norteadores da evolução individual, particularmente frente a sujeitos submetidos a intervenções.

(d) Bayley Scales of Infant Development III (Bayley): é uma escala aplicável a crianças de 1 a 42 meses de idade, fornecendo um escore de desenvolvimento intelectual que é considerado equivalente ao futuro escore de índice de inteligência - QI. Os escores estabelecidos pela Escala Bayley têm se mostrado equiparáveis aos testes padronizados de inteligência destinados a crianças maiores de cinco anos, mostrando-se um bom preditor de QI (Eldevik et al., 2009). Uma das áreas mais importantes para a avaliação da independência e melhor prognóstico de 
crianças com TEA, se refere ao seu desenvolvimento intelectual, por isso essa medida foi incluída no presente estudo.

\section{Fase 3}

Além dos instrumentos aplicados na fase 2, nessa terceira fase foram realizadas também avaliações médicas: uma avaliação clínica das 11 crianças por um médico geneticista e especialista em TEA, com base nos critérios do Manual Diagnóstico e Estatístico de Transtornos Mentais (American Psychiatric Association, 2000), com o objetivo de estabelecer o diagnóstico de TEA ou descartar o transtorno.

\section{Procedimentos}

Em 2010, as famílias das quatro crianças que tiveram sinais precoces de TEA detectados segundo M-CHAT na pesquisa de 2009 , assim como as famílias dos oito controles, foram contatadas para novas avaliações por meio da Secretaria da Educação de Barueri. As avaliações das crianças foram realizadas em salas privativas das creches (com exceção de uma criança que havia saído da creche), enquanto as mães foram entrevistadas em casa ou na própria creche dependendo de sua disponibilidade. As avaliações médicas foram realizadas na creche, em sala previamente designada pela coordenação, havendo o cuidado de minimizar o impacto da presença dos pesquisadores na rotina das crianças e dos funcionários de cada uma das creches.

O OERA foi realizado por meio de gravações de vídeos estruturados de aproximadamente 10 minutos que posteriormente foram analisados por duas psicólogas especialistas na área de autismo, para avaliar o nível de risco para TEA dos casos. Considerando aspectos das provas específicas em conjunto com a avaliação geral, as crianças foram classificadas pelas especialistas como provável caso de TEA ou como criança com desenvolvimento típico. As duas psicólogas eram completamente neutras ("cegas") quanto: (i) aos objetivos do estudo, (ii) quanto a condição das crianças avaliadas, ou seja, se eram de alto risco ou de baixo risco para TEA, (iii) assim como quanto a pontuação que estava sendo fornecida por sua colega. O critério de concordância entre examinadores foi dado em relação à pontuação total do OERA, assim como em relação ao desfecho final fornecido pelo instrumento: provável caso de TEA ou desfecho negativo para TEA. Assim, o critério de concordância entre examinadores foi emitido por consenso.

\section{Considerações éticas}

Todas as avaliações foram realizadas após o consentimento por escrito das mães ou responsáveis, mantendo-se o sigilo das informações e o anonimato dos participantes. Além disso, um termo de imagem foi assinado por todas as mães quando seus filhos ingressaram nas creches em que a pesquisa foi realizada, sendo assim permitida a utilização das imagens das crianças nas filmagens desta pesquisa. Esse estudo foi aprovado pelo Comitê de Ética em Pesquisa com Seres Humanos da instituição de ensino em que foi realizado, sob o protocolo $\mathrm{n}^{\circ}$ 0005.0.272.000-09.

Ao final da pesquisa foi realizada uma devolutiva a comunidade, por meio de palestras sobre atrasos de desenvolvimento neuropsicomotor para os profissionais das creches envolvidas. Para os pais das quatro crianças com suspeita de TEA também foi feita uma devolutiva individual de acordo com o desfecho do estudo na terceira fase.

\section{RESULTADOS}

Entre a $2^{\mathrm{a}}$ e a $3^{\mathrm{a}}$ fase do estudo foi possível localizar 11 das 12 crianças da amostra, três com suspeita de TEA e oito sem suspeita de TEA, já que uma das crianças mudou de cidade neste período e por mais que tivéssemos solicitado uma entrevista, os familiares não aceitaram. Dos 11 casos avaliados nestas duas novas fases, verificou-se que nenhum deles confirmou diagnóstico de TEA após completarem três anos de idade, segundo o diagnóstico médico realizado pelo geneticista da equipe, assim como de acordo com instrumento $\mathrm{ABC}$.

Como esperado, o OERA foi sensível em identificar o desenvolvimento típico das 11 crianças, ou seja, não houve diferenças na pontuação do protocolo de observação estruturada entre os três casos com suspeita de TEA e os oito sem suspeita de TEA, tendo todos eles pontuado entre zero e dois. Contudo, não foi possível estabelecer o ponto de corte do OERA, já que todas as crianças negativaram para TEA. As observadoras independentes (duas psicólogas experientes na assistência a crianças com TEA) concordaram em $100 \%$ quanto ao desfecho negativo das 11 crianças, enquanto sutis variações (de um a dois pontos) foram verificadas no cômputo total do OERA.

A fim de descrever mais detalhadamente o perfil clínico e comportamental de cada um dos casos classificados como suspeitos de TEA segundo o M-CHAT e aprofundar a compreensão dos aspectos em que apresentavam atrasos no desenvolvimento (que futuramente se mostraram não específicos para os TEA), abaixo apresentaremos os resultados nos testes obtidos por cada um deles. 


\section{Caso 1 - A., gênero feminino.}

Aos 20 meses, foi aplicado o instrumento de rastreamento M-CHAT (tendo as cuidadoras das creches como informantes) cujo resultado foi "risco elevado para TEA", identificado pela falha em um único ponto crítico " $<$ nome da criança $>$ já usou o dedo indicador dele para apontar, para pedir alguma coisa?”. Em contrapartida, aos 42 meses, foi aplicado o ABC, cujo ponto de corte é 47, e apontou "negativo para TEA", obtendo um total de 35 pontos, abaixo do esperado para ser considerado como "leve probabilidade de Autismo". Embora A. tenha pontuado positivo em alguns comportamentos indicativos de sinais para TEA, como uso inadequado de pronomes e hábito de repetir perguntas, o déficit no item "não apontar para pedir alguma coisa", anteriormente identificado pelo, M-CHAT não se manteve com o passar do tempo, indicando falhas temporárias no desenvolvimento, o que não é compatível com o quadro de TEA. Na primeira avaliação da Bayley, A. apresentou escore médio inferior no domínio da linguagem. Ressalta-se que na segunda avaliação a criança já havia atingido a idade máxima de 42 meses admitida pela escala, dessa forma, fez-se uma leitura qualitativa dos resultados e embora A. tenha se apresentado globalmente dentro do desenvolvimento normal, manteve um atraso de linguagem expressiva.

Em relação à Vineland, a primeira aplicação foi feita quando A. estava com 40 meses e a segunda com 46 meses, sendo que o domínio da linguagem, prejudicado na primeira avaliação manteve-se abaixo do esperado para sua idade cronológica. O escore total da escala apontou para atraso no desenvolvimento nas duas últimas fases do estudo, sendo que foi obtida uma diferença de sete meses negativos em relação à primeira avaliação e quatro meses negativos na equivalência etária em relação à segunda aplicação, havendo ligeira melhora da primeira para a segunda avaliação.

$\mathrm{Na}$ aplicação do OERA, houve consenso entre as duas psicólogas avaliadoras tanto em relação ao desfecho negativo para TEA, quanto para a identificação de dificuldades nos domínios de interação recíproca e de linguagem. No campo linguagem, os prejuízos mais significativos foram verificados nas questões relativas à "vocalização dirigida ao outro" (baixa qualidade e frequência da vocalização dirigida socialmente) e "entoação de vocalizações ou verbalizações" (indicando anormalidades da fala, como discurso lento e/ou hesitante). Ambos avaliadores destacaram a timidez excessiva de A. durante a gravação do vídeo, sendo que essa dificuldade, provavelmente prejudicou seu desempenho durante as avaliações.
É importante ressaltar que como A. saiu da creche após a primeira fase do estudo, é provável que não tenha tido estimulações e contatos sociais com outras crianças de sua idade o que pode ter afetado a qualidade de sua interação social, justificando seu comportamento reservado diante de pessoas desconhecidas e de uma câmera de vídeo, bem como alguns indicadores de atraso no desenvolvimento em relação às crianças de mesma idade.

Em resumo, dado o desfecho negativo para TEA avaliado pelo $\mathrm{ABC}$, pode-se inferir que esses comportamentos emitidos por A. podem ser manifestações de traços de personalidade associados à timidez, retraimento e a baixa qualidade de interação social em comparação com as crianças da mesma faixa etária avaliadas em nossa pesquisa. Dessa forma, percebe-se a importância da observação direta, demonstrado por Baranek (1999) em seus estudos, para assim poder identificar os traços de atraso de desenvolvimento característicos do TEA diferenciandoos das particularidades de cada criança e assim, analisar a situação dado o seu contexto global.

\section{Caso 2 - K., gênero masculino.}

$\mathrm{K}$. falhou em apenas um item crítico do M-CHAT (respondido pelas cuidadoras das creches), a saber: " $<$ nome da criança $>$ alguma vez trouxe objetos para você (pais) para lhe mostrar este objeto?". Neste caso, o M-CHAT não foi capaz de discriminar sintomas de TEA de sintomas ligados a um atraso mais global. Essa criança tem uma limitação física particularmente acentuada nos membros inferiores, tendo uma suspeita de paralisia cerebral do tipo diparesia espástica. As dificuldades motoras apresentadas pela criança acabaram levando, portanto, à identificação de um caso falso positivo.

$\mathrm{Na}$ segunda fase do estudo, quando tinha três anos e oito meses, $\mathrm{K}$ obteve pontuação baixa no $\mathrm{ABC}$ (sete pontos positivos), tendo sido classificado como "criança normal". Por outro lado, mesmo com uma baixa pontuação neste instrumento de rastreamento, $\mathrm{K}$ tinha alguns atrasos, como por exemplo, não conseguia se vestir sozinho, assim como problemas de comportamento como destrutividade em relação a objetos da casa, e ter o hábito de brincar girando e batendo os objetos várias vezes.

Já em relação à avaliação cognitiva, K. apresentou atraso em todos os domínios da escala Bayley na segunda fase da pesquisa: média baixa para domínio cognitivo e limítrofe para os domínios de linguagem e motor. Na terceira fase, a criança continuou apresentando atrasos nos domínios cognitivo, linguagem receptiva e expressiva e atraso expressivo nos domínios 
motores (fino e grosso), mesmo tendo excedido a idade limite das Escalas de Bayley. Esse atraso tão evidente se deu principalmente devido a sua limitação física global, pois mesmo nas provas cognitivas e de linguagem, a escala Bayley exige movimentações e comportamentos motores que provavelmente influenciaram negativamente todos os domínios de avaliação.

Em relação à Vineland, K. realizou a primeira testagem com 2 anos e 6 meses, sendo seu desempenho global correspondente ao de uma criança de 3 anos, e apresentou um desempenho equivalente a três anos de idade para as áreas de comunicação, mesmo com sua dificuldade motora. Em contrapartida, K. teve um desempenho inferior ao da sua idade na terceira fase do estudo quando ele tinha 3 anos e 7 meses. $\mathrm{Na}$ avaliação geral, ele apresentou um desempenho condizente ao de uma criança de 2 anos e 9 meses, obtendo escores bastante inferiores na área da comunicação (equivalência etária de 2 anos e 5 meses) e de habilidades motoras (equivalência etária de 1 ano e 9 meses). Faz-se necessário destacar esse retrocesso no desenvolvimento de $\mathrm{K}$. mesmo estando ele exposto às estimulações da creche. Uma possível explicação para isso é que com o passar do tempo, o nível de exigência dos testes de avaliação fica maior, e uma criança como $\mathrm{K}$. que apresenta déficits motores, vai ficando mais atrasada em relação aos pares da mesma idade.

No OERA, K. interagiu o tempo todo com o avaliador e apesar de apresentar diversos lapsos na linguagem, buscou ativamente estabelecer diálogo na maior parte da prova. Além disso, não apresentou déficits em provas bem significativas para o estabelecimento de TEA, como iniciação e resposta a atenção compartilhada, tampouco na prova do chamado ao nome. Assim, o OERA realizado quando $\mathrm{K}$. tinha 30 meses, deixou evidente que não se tratava de uma criança com TEA, mas de uma criança com déficits motores.

Em resumo, dada a alta sensibilidade do M-CHAT para identificação de transtornos globais do desenvolvimento, pode-se dizer que nesse caso em particular, mostrou-se pouco específico na identificação de TEA, pois os atrasos de $\mathrm{K}$ eram decorrentes de seus déficits motores que o M-CHAT não discriminou dos de TEA. Essa limitação do M-CHAT vai de encontro com as orientações de especialistas em TEA que referem que dados informados pelos cuidadores devem ser aliados à observação direta dos comportamentos da criança (Ozonoff et al., 2008), que o OERA captou bem na presente pesquisa.

\section{Caso 3 -S., Gênero: feminino.}

O M-CHAT foi respondido pela cuidadora da creche quando S. tinha 24 meses. A criança foi con- siderada com risco para TEA devido à identificação de problemas em três itens, sendo todos eles não graves: "O (a) <nome da criança> gosta de subir em coisas como, escadas ou móveis?", " $<\mathrm{O}$ (a) nome da criança $>$ já pareceu muito sensível ao barulho (ex: tapando os ouvidos)?" e "O (a) < nome da criança $>$ faz movimentos estranhos com os dedos perto do rosto dele". Aos 50 meses, segundo o instrumento ABC, S. teve pontuação 15 sendo considerada sem risco para TEA. Porém, é interessante ressaltar, que os dois sintomas anteriormente identificados pelo M-CHAT foram confirmados no ABC: "Balança as mãos" e "Tapa as orelhas para vários sons". Estes sintomas são incomuns em crianças com desenvolvimento típico e mereceriam uma investigação mais criteriosa da criança, a fim de descartar um possível caso de TEA. Se por um lado sabemos que apenas três sintomas não permitem a classificação de TEA, por outro, diferentemente das outras duas crianças relatadas acima, os sintomas de S. parecem mais ligados a este quadro clínico, principalmente pelo fato destes terem permanecido estáveis aos quatro anos de idade. Como $\mathrm{S}$. foi avaliada por um médico especialista em TEA e o diagnóstico deste foi excluído, é possível que estes sinais sejam indicativos de outras condições ainda não explicadas pela avaliação do presente estudo. Outra suposição é que um comportamento normal ou decorrente de outro aspecto do desenvolvimento tenha sido superestimado pela mãe que foi quem respondeu o ABC. Por exemplo, ao analisar em detalhes o protocolo de $\mathrm{S}$. verificamos que no item "Balança as mãos" do $\mathrm{ABC}$, havia uma observação do entrevistador de que apesar de presente, este sintoma se restringia apenas aos momentos, que segundo a mãe, $\mathrm{S}$ estaria fazendo birra. De qualquer modo, $\mathrm{S}$, pontuou negativamente na $2^{\text {a }}$ fase do estudo, em todos os instrumentos relativos à TEA (ABC, OERA e avaliação médica segundo critérios do DSM-IV).

Em relação ao teste Bayley, na primeira aplicação, com 41 meses, a criança apresentou média baixa para o domínio linguagem. Na segunda testagem, S conseguiu realizar as provas propostas pela Bayley apresentando algumas falhas nas provas de linguagem, contudo, sua idade havia ultrapassado o limite de 42 meses deste teste. Por meio de uma análise qualitativa, é possível afirmar que globalmente S. se encontra dentro da faixa da normalidade na avaliação da Escala Bayley III, mesmo que aspectos da linguagem apareçam moderadamente comprometidos.

$\mathrm{Na}$ primeira aplicação da Vineland, S. estava com 38 meses e apresentou atrasos evidentes nos domínios da comunicação (desempenho equivalente a uma criança de 18 meses), sendo que este atraso foi 
atenuado na terceira fase quando ele tinha 50 meses de idade e desempenho correspondente ao de uma criança de 44 meses. Na avaliação global da Vineland, a criança apresentou atraso, sendo seu desempenho correspondente ao de uma criança de 45 meses. Assim, S continuou com algum atraso no domínio da comunicação, mas com melhoras expressivas entre as últimas duas fases do estudo. Conclui-se com isso, que $\mathrm{S}$. apresenta certo atraso na linguagem que merece estimulação, mas quando analisado o comportamento global da criança, percebe-se que este atraso não se encaixa no perfil de TEA, já que outras áreas do desenvolvimento não estão comprometidas.

Em relação ao OERA, realizada aos 34 meses o avaliador 1, identificou dois comportamentos compatíveis com sintomas de TEA, ambos na área da linguagem e comunicação. A primeira delas foi uma falha na "frequência da vocalização dirigida ao outro" a segunda, uma inadequação na "entonação de vocalizações ou verbalizações". Nota-se que ambas as provas apontam para uma limitação de S. em procurar o examinador para estabelecer uma conversa, seja ela amigável ou para exprimir suas necessidades, além de pouca variação do tom, com a fala tendendo a ser lenta e monótona. Já o avaliador 2 não identificou sinais de TEA, pontuando um total de zero nesta avaliação. Mesmo que o avaliador 1 tenha identificado dois sintomas de TEA, ambos refutaram o diagnóstico geral deste transtorno e citaram a timidez como um dos fatores que influenciaram negativamente seu desempenho nas provas. Ao analisar o vídeo, o padrão comportamental de timidez é evidente, indicando que as falhas de S. são predominantemente desta natureza e não refletem um perfil apresentado por crianças com TEA.

Em resumo, essa criança apresenta déficits na área da comunicação que se mostram estáveis no decorrer das fases da pesquisa, tanto segundo a informação dos pais quanto na observação da criança (OERA). O domínio da comunicação é um dos comprometidos na área dos TEA e pode ter sido o motivo para que essa criança tenha sido classificada precocemente como risco para TEA, mesmo que este diagnóstico não tenha sido comprovado. Neste caso, o instrumento OERA foi o mais eficaz para discriminar timidez com déficits na linguagem de um quadro TEA, apontando para a importância da observação direta dos casos (Baranek, 1999; DiLavore, Lord, \& Rutter, 1995), assim como para mais uma evidência de eficácia do OERA.

\section{Caso 4 - E., masculino.}

Mudou-se de cidade e a família se recusou a continuar a participar do estudo, por isso foi considerado perda amostral.

Os principais resultados do estudo encontram-se sintetizados na Tabela 1, a seguir.

TABELA 1

Caracterização de desempenho das crianças com alto risco de desenvolverem TEA, segundo instrumentos de avaliação utilizados nas três fases do estudo.

\begin{tabular}{|c|c|c|c|c|c|c|}
\hline Identificação & $\begin{array}{c}\text { M-CHAT } \\
\text { Fase } 1\end{array}$ & $\begin{array}{c}A B C \\
\text { Fases 2-3 }\end{array}$ & $\begin{array}{c}\text { BAYLEY } \\
\text { Fases 2-3 }\end{array}$ & $\begin{array}{l}\text { VINELAND } \\
\text { Fases 2-3 }\end{array}$ & $\begin{array}{c}\text { OERA } \\
\text { Fases 2-3 }\end{array}$ & $\begin{array}{l}\text { Especificidades } \\
\text { do caso }\end{array}$ \\
\hline $\begin{array}{l}\text { Caso } 1 \\
\text { Feminino }\end{array}$ & $\begin{array}{l}\text { Risco elevado } \\
\text { para TEA: } \\
\text { falhou em um } \\
\text { item crítico }\end{array}$ & $\begin{array}{l}\text { Negativo } \\
\text { para TEA }\end{array}$ & $\begin{array}{l}\text { Escore médio inferior } \\
\text { no domínio de } \\
\text { linguagem expressiva }\end{array}$ & $\begin{array}{l}\text { Domínio da } \\
\text { linguagem abaixo } \\
\text { do esperado para a } \\
\text { idade }\end{array}$ & $\begin{array}{l}\text { Dificuldades } \\
\text { nos domínios de } \\
\text { interação recíproca } \\
\text { e de linguagem. } \\
\text { Avaliadores } \\
\text { destacaram a timidez } \\
\text { excessiva }\end{array}$ & $\begin{array}{l}\text { Indicadores } \\
\text { comportamentais } \\
\text { de timidez e } \\
\text { retraimento }\end{array}$ \\
\hline $\begin{array}{l}\text { Caso } 2 \\
\text { Masculino }\end{array}$ & $\begin{array}{l}\text { Risco elevado } \\
\text { para TEA: } \\
\text { falhou em um } \\
\text { item crítico }\end{array}$ & $\begin{array}{l}\text { Negativo } \\
\text { para TEA }\end{array}$ & $\begin{array}{l}\text { Atrasos nos domínios } \\
\text { cognitivo, linguagem } \\
\text { receptiva/ expressiva; } \\
\text { e motores fino/grosso }\end{array}$ & $\begin{array}{l}\text { Déficits nas áreas da } \\
\text { comunicação e de } \\
\text { habilidades motoras }\end{array}$ & $\begin{array}{l}\text { Sem déficits em } \\
\text { provas de TEA; } \\
\text { indicadores de } \\
\text { déficits motores }\end{array}$ & $\begin{array}{l}\text { Limitação física } \\
\text { evidente decorrente } \\
\text { de suspeita de } \\
\text { paralisia cerebral }\end{array}$ \\
\hline $\begin{array}{l}\text { Caso } 3 \\
\text { Feminino }\end{array}$ & $\begin{array}{l}\text { Risco elevado } \\
\text { para TEA: } \\
\text { falhou em um } \\
\text { item crítico }\end{array}$ & $\begin{array}{l}\text { Negativo } \\
\text { para TEA }\end{array}$ & $\begin{array}{l}\text { Na faixa da } \\
\text { normalidade; } \\
\text { alguns aspectos da } \\
\text { linguagem afetados }\end{array}$ & $\begin{array}{l}\text { Atraso no domínio } \\
\text { da comunicação, } \\
\text { mais atenuado na } \\
\text { fase } 3\end{array}$ & $\begin{array}{l}\text { Sem déficits em } \\
\text { provas de TEA; } \\
\text { indicadores de } \\
\text { dificuldades } \\
\text { emocionais e } \\
\text { comportamentais } \\
\text { como timidez } \\
\text { interferindo no } \\
\text { desempenho nas } \\
\text { provas }\end{array}$ & $\begin{array}{l}\text { Indicadores } \\
\text { comportamentais } \\
\text { de timidez e } \\
\text { retraimento com } \\
\text { comprometimento } \\
\text { na comunicação }\end{array}$ \\
\hline $\begin{array}{l}\text { Caso } 4 \\
\text { masculino }\end{array}$ & - & - & - & - & - & - \\
\hline
\end{tabular}


Um dos achados mais importantes do estudo foi verificar que os resultados do OERA analisados por duas psicólogas foram $100 \%$ compatíveis com $\mathrm{o}$ instrumento de rastreamento $\mathrm{ABC}$ e da avalição médica com o DSM-IV, ou seja, as 11 crianças da amostra foram consideradas com desenvolvimento típico. Este resultado indica que no grupo estudado o OERA mostrou-se eficaz para discriminar as crianças que não preencheram o diagnóstico de TEA de acordo com o DSM-IV (American Psychiatric Association, 2000). Entretanto, pelo fato de nenhuma delas ter fechado diagnóstico de TEA, ao final do estudo não foi possível confirmar se o instrumento também é eficaz para identificar casos positivos de TEA. Essa é uma limitação da presente pesquisa, e novos estudos com a participação de crianças com diagnóstico pré-confirmado de TEA são recomendáveis.

$O$ fato de nenhum dos casos ter fechado diagnóstico de TEA indica que o M-CHAT mostrou-se um instrumento sensível para identificar precocemente atrasos globais do desenvolvimento, mas pouco específico para identificar TEA; ou seja, apesar de ser um instrumento muito útil na avaliação de atrasos globais do desenvolvimento, deve ser usado com cautela no rastreamento de sinais precoces de TEA.

\section{CONCLUSÕES}

Se por um lado a identificação precoce é uma medida de alta relevância, pois permite intervenções precoces, levando ao melhor prognóstico dos casos de TEA, por outro lado, é preciso cautela com um diagnóstico errôneo baseado em um único instrumento de rastreamento. Esse estudo mostrou que o M-CHAT identificou atrasos globais e alguns sinais críticos. No entanto, a não confirmação do diagnóstico nas fases 2 e 3 mostra que o instrumento é sensível a determinados sinais do transtorno, mas pouco específico no que concerne ao TEA.

Recomenda-se que o rastreamento de suspeitas de atraso de desenvolvimento ou sinais de TEA em crianças até aproximadamente três anos de idade seja feito com a utilização de múltiplos informantes concomitantemente à observação da própria criança, a fim de garantir maior acurácia na seleção para tomar as providências necessárias e poder garantir melhores diagnósticos e prognósticos (Gray \& Tonge, 2005). Estudos recentes que discutem o uso de diretrizes clínicas para o diagnóstico de TEA recomendam que, em crianças de um a sete anos de idade sob suspeita de TEA, sejam utilizadas múltiplas ferra- mentas de avaliação em monitoramentos periódicos para esclarecer se os sinais/sintomas medidos por elas são ou não preditores do futuro diagnóstico de TEA (Hathorn, Alateegi, Graham, \& O'Hare, 2014).

Um diagnóstico de TEA exige cautela em relação ao uso de instrumentos e, posteriormente no que concerne à devolutiva aos familiares e profissionais envolvidos, deixando claro que atrasos precoces devem ser avaliados mais acuradamente antes de serem considerados problemas mais sérios compatíveis com TEA. Nesse sentido o presente estudo pode ser tomado como modelo em unidades de educação infantil já que permitiu não só verificar sinais de TEA como de outros atrasos de desenvolvimento, segundo avaliações em momentos distintos do desenvolvimento infantil. O estudo teve a limitação de trabalhar como um grupo amostral pequeno desde a primeira fase do estudo formado por 104 crianças, entretanto considera-se expressivo que em quatro delas tenha sido constatada a suspeita para TEA. Adicionalmente, aponta-se que os instrumentos de rastreamento de sinais precoces de TEA foram preenchidos por diferentes cuidadoras em cada uma das etapas do estudo, sendo esta uma fragilidade metodológica, que procuramos minimizar, tendo como critérios de inclusão cuidadoras que (i) tinham no mínimo um ano de contato com a criança em avaliação e (ii) que se declararam capazes de responder às questões dos questionários.

A análise de vídeos tem sido uma ferramenta bastante utilizada internacionalmente para comparar o desenvolvimento de indivíduos ao longo do tempo. O protocolo estruturado para análise de vídeo, OERA, desenvolvido por nossa equipe, mostrou-se uma técnica acessível do ponto de vista econômico, bem aceita nas creches do município estudado e importante para a discriminação entre timidez e déficits na sociabilidade/comunicação, apresentando-se como uma estratégia adequada para avaliação do desenvolvimento infantil adaptada à realidade brasileira. Essas evidências preliminares indicam um bom potencial do protocolo como uma estratégia de triagem para descartar crianças com desenvolvimento típico de apresentarem TEA. Entretanto, na amostra estudada, nenhuma das crianças com sinais precoces fechou diagnóstico de TEA entre três e cinco anos de idade, o que limitou a compreensão sobre os alcances do OERA. Deste modo, recomenda-se a realização de novos estudos, com amostras mais robustas, incluindo crianças com TEA previamente diagnosticado, o que permitirá a comparação dos resultados com o de crianças com desenvolvimento 
típico e consequentemente, o estabelecimento de medidas de validade do OERA. Futuros estudos de evidências de validade do OERA, permitirão o estabelecimento de indicadores de sensibilidade e especificidade desse instrumento, importante estratégia para validar quantitativamente a capacidade de discriminação do modelo de avaliação utilizado no presente estudo.

\section{REFERÊNCIAS}

American Psychiatric Association (APA). (2000). Diagnostic and Statistic Manual of Mental Disorders - Text Revision (DSM-IVTR) (4 $4^{\text {th }}$ ed.). Washington.

American Psychiatric Association (APA). (2013). Diagnostic and Statistic Manual of Mental Disorders (DSM-V) ( $5^{\text {th }}$ ed.). Washington.

Baranek, G. T. (1999). Autism during infancy: a retrospective video analysis of sensory-motor and social behaviors at 9-12 months of age. Journal of Autism and Developmental Disorders, 29(3), 213-224. doi: 10.1023/A:1023080005650.

Belini, A. E. G. \& Fernandes, F. D. M. (2007). Olhar de bebês em desenvolvimento típico: correlações longitudinais encontradas. Revista da Sociedade Brasileira de Fonoaudiologia, 12(3), 165-173. doi: 10.1590/S1516-80342007000300003.

Becker, M. M., Wagner, M. B., Bosa, C. A., Schmidt, C., Longo, D., Papaleo, C., \& Riesgo, R. S. (2012). Translation and validation of Autism Diagnostic Interview-Revised (ADI-R) for autism diagnosis in Brazil. Arquivos de Neuro-psiquiatria, 70(3), 185190. doi: 10.1590/S0004-282X2012000300006.

Bosa, C. A. (2006). Autismo: intervenções psicoeducacionais. Revista Brasileira de Psiquiatria, 28(1), 47-53. doi: 10.1590/ S1516-44462006000500007.

Cardoso, C. \& Fernandes, F. D. M. (2006). Relação entre os aspectos sócio cognitivos e perfil funcional da comunicação em um grupo de adolescentes do espectro autístico. Pró-Fono Revista de Atualização Científica, 18(1), 89-98.

Carvalho, F. A., Paula, C. S., Teixeira, M. C. T. V., Zaqueu, L. D. C. C., \& D'Antino, M. E. F. (2013). Rastreamento de sinais precoces de transtorno do espectro do autismo em crianças de creches de um município de São Paulo. Revista Psicologia: Teoria e Prática, 15(2), 144-154.

Clifford, S., Young, R., \& Williamson, P. (2007). Assessing the early characteristics of autistic disorder using video analysis. Journal of Autism and Developmental Disorders, 37(2), 301313. doi: 10.1007/s10803-006-0160-8.

Delgado, C., Mundy, P., \& Block, J. (2001). Pictorial infant communication scale (PICS) version 1.3. Coral Gables: University of Miami.

DiLavore, P. C., Lord, C., \& Rutter, M. (1995). The pre-linguistic autism diagnostic observation schedule. Journal of Autism and Developmental Disorders, 25(4), 355-379. doi: 10.1007/ BF02179373.

Eldevik, S., Hastings, R. P., Hughes, J. C., Jahr, E., Eikeseth, S., \& Cross, S. (2009). Meta-Analysis of Early Intensive Behavioral Intervention for Children with Autism. Journal of Clinical Child and Adolescent Psychology: The Official Journal for the Society of Clinical Child and Adolescent Psychology, American Psychological Association, Division 53, 38(3), 439-450. doi: 10.1080/15374410902851739.
Elsabbagh, M., Divan, G., Koh, Y. J., Kim, Y. S., Kauchali, S., Marcín, C., \& Fombonne, E. (2012). Global prevalence of autism and other pervasive developmental disorders. Autism Research, 5(3), 160-179. doi: 10.1002/aur.239.

Fombonne, E. (2009). Epidemiology of pervasive developmental disorders. Pediatric Research, 65, 591-598. doi:10.1203/pdr. 0b013e31819e7203.

Gray, K. M. \& Tonge, B. J. (2005). Screening for autism in infants and preschool children with developmental delay. Australian and New Zealand Journal of Psychiatry, 39(5), 378-386. doi: 10.1111/j.1440-1614.2005.01585.x.

Gotham, K., Pickles, A., \& Lord, C. (2009). Standardizing ADOS scores for a measure of severity in autism spectrum disorders. Journal of autism and developmental disorders, 39(5), 693-705. doi: 10.1007/s10803-008-0674-3.

Hathorn, C., Alateeqi, N., Graham, C., \& O'Hare, A. (2014). Impact of Adherence to Best Practice Guidelines on the Diagnostic and Assessment Services for Autism Spectrum Disorder. Journal of autism and developmental disorders, 1-8. doi: 10.1007/s10803014-2057-2.

Kalb, L. G., Law, J. K., Landa, R., \& Law, P. A. (2010). Onset patterns prior to 36 months in autism spectrum disorders. Journal of Autism and Developmental Disorders, 40(11), 13891402. doi: 10.1007/s10803-010-0998-7.

Kleinman, J. M., Robins, D. L., Ventola, P. E., Pandey, J., Boorstein, H. C., Esser, E. L., \& Fein, D. (2008). The modified checklist for autism in toddlers: a follow-up study investigating the early detection of autism spectrum disorders. Journal of Autism and Developmental Disorders, 38(5), 827-839. doi: 10.1007/s10803007-0450-9.

Krug D. A., Arick J., \& Almond P. (1980). Behavior checklist for identifying severely handicapped individuals with high levels of autistic behavior. Journal of the American Academy of Child \& Adolescent Psychiatry, 21(3), 221-9. doi: 10.1111/j.14697610.1980.tb01797.x.

Ozonoff, S., Young, G. S., Goldring, S., Greiss-Hess, L., Herrera, A. M., Steele, J., \& Rogers, S. J. (2008). Gross motor development, movement abnormalities, and early identification of autism. Journal of Autism and Developmental Disorders, 38(4), 644656. doi: 10.1007/s10803-007-0430-0.

Ozonoff, S., Iosif, A. M., Baguio, F., Cook, I. C., Hill, M. M., Hutman, T., \& Young, G. S. (2010). A prospective study of the emergence of early behavioral signs of autism. Journal of the American Academy of Child and Adolescent Psychiatry, 49(3), 256-266. doi:10.1016/j.jaac.2009.11.009.

Paula, C. S., Ribeiro, S. H., Fombonne, E., \& Mercadante, M. T. (2011). Brief report: Prevalence of pervasive developmental disorder in Brazil: A pilot study. Journal of Autism and Developmental Disorders, 41(12), 1738-1742. doi: 10.1007/ s10803-011-1200-6.

Robins, D. L., Fein, D., Barton, M. L., \& Green, J. A. (2001). The Modified Checklist for Autism in Toddlers: an initial study investigating the early detection of autism and pervasive developmental disorders. Journal of Autism and Developmental Disorders, 31(2), 131-144. doi: 10.1023/A: 1010738829569.

Sparrow, S. S. \& Cicchetti, D. V. (1985). Diagnostic uses of the vineland adaptive behavior scales. Journal of Pediatric Psychology, 10(2), 215-225. doi: 10.1093/jpepsy/10.2.215.

Tamanaha, A. C., Perissinoto, J., \& Chiari, B. M. (2008). Evolução da criança autista a partir da resposta materna ao Autism Behavior Checklist. Pró-Fono, 20(3), 165-70. 
Teixeira, M. C. T. V., Mecca, T. P., Velloso, R. L., Bravo, R. B., Ribeiro, S. H. B., Mercadante, M. T., \& Silvestre, C. (2010). Literatura científica brasileira sobre transtornos do espectro autista. Revista da Associação Médica Brasileira, 56(5), 607614. doi: 10.1590/S0104-42302010000500026.

Zwaigenbaum, L. (2010). Advances in the Early Detection of Autism. Current Opinion in Neurology, 23(2), 97-102.

\section{Autores:}

Felipe Alckmin-Carvalho - Mestre em Psicologia Clínica pela Universidade de São Paulo, Professor do Departamento de Saúde III - Universidade Nove de Julho.

Maria Cristina Triguero Veloz Teixeira - Professora Adjunta do Programa de Pós-Graduação em Distúrbios do Desenvolvimento da Universidade Presbiteriana Mackenzie.
Décio Brunoni - Professor Titular do Programa de Pós-Graduação em Distúrbios do Desenvolvimento da Universidade Presbiteriana Mackenzie.

Vanessa Godoy Strauss - Graduada pela Universidade Presbiteriana Mackenzie. Cristiane Silvestre Paula - Professora Adjunta do Programa de Pós-Graduação em Distúrbios do Desenvolvimento da Universidade Presbiteriana Mackenzie.

Endereço para correspondência:

Felipe Alckmin-Carvalho

Universidade de São Paulo

Instituto de Psicologia - Departamento de Psicologia Clínica

Av. Professor Mello Moraes, 1721

CEP 05508030 São Paulo, SP, Brasil

Recebido em: $16 / 11 / 2013$

Aceito em: 15/04/2014 